\title{
Coexistence of aortic valve stenosis and cardiac amyloidosis: echocardiographic and clinical significance
}

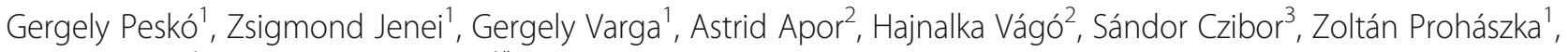
Tamás Masszi ${ }^{1}$ and Zoltán Pozsonyi ${ }^{*}$ (I)

\begin{abstract}
Background: Left ventricular hypertrophy and diastolic dysfunction are common echocardiographic features of both aortic valve stenosis (AS) and cardiac amyloidosis (CA). These two different entities therefore may mask each other. From recent years, there is a growing body of evidence about the relatively high incidence of wild-type transthyretin (wtTTR) amyloidosis in AS, but there are scarce data on the prevalence of AS in CA, particularly in ALtype amyloidosis. The echocardiographic approach to these patients is not obvious, and not evidence based. We aimed to study the prevalence, severity, and type of AS in patients with CA and also to evaluate the potential of echocardiography in the diagnostic process.
\end{abstract}

Methods: Between January 2009 and January 2019, we retrospectively analyzed the clinical and echocardiographic data, and the echocardiographic work up of 55 consecutive CA patients.

Results: $80 \%$ of our CA patients had AL amyloidosis. We identified 5 patients (9\%) with moderate to severe AS: two with moderate AS and three with low-flow, low-grade AS (LFLG AS). Further analysis of the latter three patients with dobutamine stress echocardiography revealed pseudo-severe LFLG AS in two, and true-severe AS in one patient.

Conclusion: The prevalence of moderate to severe AS is $9 \%$ in our population of CA patients, the majority of whom have AL amyloidosis. Dobutamine echocardiography seems to be appropriate for the further characterization of patients with LFLG AS, even with normal ejection fraction.

Keywords: Cardiac amyloidosis, Aortic valve stenosis, Dobutamine stress echo

\section{Introduction}

Aortic valve stenosis (AS) is a common valvular heart disease, which significantly reduces life expectancy when it becomes symptomatic [1]. The prevalence of AS is increasing with age, becoming as high as 12.4 and $3.4 \%$ for severe AS in a patient population aged more than 75 years, according to a recent metaanalysis [2]. Cardiac amyloidosis (CA) is generally considered a rare condition caused by protein deposition in the heart. The two most common forms are monoclonal light chain amyloidosis (AL), and transthyretin (TTR) CA [3]. In the

\footnotetext{
* Correspondence: pozsonyizoltanimre@gmail.com

13rd Department of Internal Medicine, Semmelweis University, Kútvölgyi 4,

Budapest 1125, Hungary

Full list of author information is available at the end of the article
}

former, plasma cell dyscrasia (PCD) results in the production of monoclononal light chains, which infiltrate the heart. In wild-type TTR (wtTTR) amyloidosis seen in elderly patients, the wild type of TTR is responsible for the beta-sheet structure of the protein deposition. Mutant TTR (mTTR) may also infiltrate the heart; this form of the disease becomes symptomatic in the 6th or 7th decades of life.

AS and CA share common echocardiographic features, such as left ventricular hypertrophy, diastolic dysfunction, and - in many cases - elevated left ventricular filling pressure. Echocardiography may not reveal $\mathrm{CA}$ in AS, because the typical signs of CA may be readily explained by the AS. In recent years, a few case studies reported the coexistence of wtTTR cardiac amyloidosis

(c) The Author(s). 2019 Open Access This article is distributed under the terms of the Creative Commons Attribution 4.0 International License (http://creativecommons.org/licenses/by/4.0/), which permits unrestricted use, distribution, and 
and AS, highlighting the diagnostic, as well as the therapeutic challenges of these cases $[4,5]$. The studies conducted in AS patients [6-10] to examine the prevalence of TTR amyloidosis in severe AS used diverse diagnostic methods and evaluated different study populations. Some of these studies [6, 7, 9] included more than 100 AS patients. Remarkably, the incidence of TTR CA was as high as 6 to $16 \%$ in patients with AS. This question can also be examined the other way around, by determining the prevalence of AS among CA patients. Among TTR CA patients, the prevalence of moderate to severe AS was found to be as high as $27 \%$ [11]. However, the prevalence of AS in consecutive CA patients - including those with TTR and AL - has not been examined yet.

We aimed to study the prevalence, severity, and type of aortic valve stenosis in patients with CA. We also aimed to examine the potential role of dobutamine stress echocardiography in the diagnostic work up of patients with CA and low flow-low grade AS (LFLG AS).

\section{Materials and methods}

We performed a systematic review of CA patients treated in our department, between 2009 January and 2019 January, focusing on the presence of AS. We diagnosed AL-type amyloidosis when all the following criteria were met: the presence of monoclonal light chain in the serum and in the urine; the presence of plasma cell dyscrasia in bone marrow biopsy; and positive tissue biopsy confirming light chain amyloid deposition in any organ. Cardiac involvement was diagnosed when cardiac MRI showed late gadolinium enhancement as a typical sign of CA, echocardiography revealed characteristic abnormalities, or the presence of $\mathrm{AL}$ amyloid was detected in cardiac tissue by biopsy, performed either during right heart catheterization or at autopsy. Since June 2016, if no monoclonal light chain was present in the serum, we established the diagnosis of TTR CA after a positive Tc-99-pyrophosphate (PYP) isotope scan (Perugini score 2 or 3 ), specific for the disease [12]. In previous years, positive immunohistology for TTR in tissue biopsy, and the absence of monoclonal light chain in the serum was necessary for diagnosis, along with typical cardiac imaging results. We performed TTR gene sequencing in all TTR cases.

Unless it was contraindicated, we routinely performed CMR (Philips Achieva, 1,5 T, Philips, Amsterdam, The Netherlands) for differential diagnostic purposes, and as a test to confirm CA. Resting echocardiography was performed in accordance with the topical guidelines (Philips iE33, Philips, Amsterdam, The Netherlands) [13-15]. After 2015 for patients with suspicion of CA we performed left ventricular longitudinal strain analysis in order to examine segmental differences, and looked for "apical sparing". QLab 10.5 cardiovascular ultrasound quantification software was used (Philips, Amsterdam,
The Netherlands) for all strain analysis. Following the method of Phelan et his al., [16] the difference of regional left ventricular longitudinal strain (LS) between the apical and other segments of the left ventricle was examined on the bull's eye image, and calculated with the following equation: Relative apical LS = average apical LS/(average basal LS + average mid LS).

During echocardiography, the suspicion of AS was raised by valve morphology in agreement with the criteria set out in the latest European guideline on valvular heart disease [1]. We performed all the routine echocardiographic measurements for AS. When the indexed aortic valve area was less than $0.6 \mathrm{~cm}^{2}$, but low-flow, low-grade AS (LFLG AS) was diagnosed by echography at rest, we performed dobutamine stress testing to differentiate between pseudo- and true-severe AS, regardless of the left ventricular ejection fraction.

Since most of the variables exhibited skewed distributions, the descriptive statistics are presented as medians with interquartile ranges (IQR), or as percentages. The strength of the associations was calculated with the nonparametric Mann-Whitney test or the chi-square test, as appropriate.

\section{Results}

Between January 2009 and January 2019 we performed resting transthoracic echocardiography in 55 patients with cardiac amyloidosis: 44 had AL-, 9 TTR (6 with mTTR and 3 with wtTTR), and one patient had AA amyloidosis. Their median age was 65 years.

Here we summarize the most important therapeutic and mortality data of this patient population: Specific treatment of the AL patients for the plasma cell dyscrasia was driven by hematologists. In most of the cases, a combination of cyclophosphamide-bortezomib and corticosteroid was introduced first, but the whole spectrum of multiple myeloma medication was used, including lenalidomide, thalidomide, daratumumab and melphalan. Doxycylin was used in the last 4 to 5 years. Two patients had autologous bone marrow transplantation.

wtTTR patients did not receive any specific treatment. Four out of the six mTTR patients received $20 \mathrm{mg}$ tafamidis, daily. Generally, our CA patients had no ICD, and none of these 55 patients had heart transplantation.

Calculation of mortality rate is not simple, because the follow up period is no more than nine month in the cases of the latest included patients. We think, the most correct way to describe mortality in this case, is to describe the nine month mortality, which was $23 / 55$ (42\%) for the whole CA patient population.

The systematic review identified 5 patients with moderate or severe aortic valve stenosis. Important clinical, biomarker, and echocardiographic characteristics of the patients are shown in Table 1. All 5 patients with AS 
Table 1 Clinical characteristics, serum levels of cardiac biomarkers, and the main echocardiographic parameters of the 55 CA patients, grouped according to the presence or absence of aortic valve stenosis

\begin{tabular}{|c|c|c|c|}
\hline & Patients without AS $(n=50)$ & Patients with AS $(n=5)$ & $p$-value \\
\hline \multicolumn{4}{|l|}{ Clinical data } \\
\hline Age (years) & $63.5(58-73)$ & $69(68-82)$ & $p=0.055$ \\
\hline Male (n, \%) & $26(52 \%)$ & $3(60 \%)$ & $p=0.553$ \\
\hline wtTTR CA (n, \%) & $2(4 \%)$ & $1(20 \%)$ & $p=0.391$ \\
\hline mTTR CA (n, \%) & $6(12 \%)$ & $0(0 \%)$ & $p=0.485$ \\
\hline AL amyloidosis ( $n, \%)$ & $40(80 \%)$ & $4(80 \%)$ & $p=0.741$ \\
\hline AA amyloidosis $(n, \%)$ & $1(2 \%)$ & $0(0 \%)$ & $p=0.909$ \\
\hline NYHA III-IV stage (n, \%) & $32(64 \%)$ & $5(100 \%)$ & $p=0.278$ \\
\hline Atrial fibrillation $(n, \%)$ & $11(22 \%)$ & $1(20 \%)$ & $p=0.312$ \\
\hline \multicolumn{4}{|l|}{ Laboratory data } \\
\hline B-type natriuretic peptide $(\mathrm{pg} / \mathrm{ml})$ & $606(234-1240)$ & $341(77-657)$ & $p=0.303$ \\
\hline Troponin T (ng/L) & $66(39-104)$ & $134(47-215)$ & $p=0.464$ \\
\hline \multicolumn{4}{|l|}{ Echocardiography } \\
\hline Left ventricular ejection fraction (\%) & $56(43-63)$ & $59(51-60)$ & $p=0.823$ \\
\hline Septal wall thickness (mm) & $16(13-18)$ & $17(13-20)$ & $p=0.578$ \\
\hline Inferior wall thickness (mm) & $15(13-17)$ & $15(13-16)$ & $p=0.780$ \\
\hline Left ventricular end diastolic diameter (mm) & $42(36-45)$ & $41(37-42)$ & $p=0.776$ \\
\hline E/e' (Average of lateral and septal e') & $20.6(16-24)$ & $18.1(16.9-20.6)$ & $p=0.241$ \\
\hline Lateral S' & $5.5(4-7)$ & $6.25(4,25-8.3)$ & $p=0.588$ \\
\hline
\end{tabular}

AS aortic valve stenosis; wtTTR wild-type transthyretin; $m$ TTR mutant-type transthyretin; NYHA New York Heart Association. Values are presented as medians with interquartile ranges (IQR), or as percentages. The strength of the associations was calculated with the nonparametric Mann-Whitney test or the chi-square test, as appropriate

had symptoms of severe heart failure, had stage III or IV symptoms according to the New York Heart Association's classification. In three patients, echocardiography was performed for suspected cardiac amyloidosis, as plasma cell dyscrasia was diagnosed before the onset of the signs of heart failure. In the two other cases echo was requested by the clinician, due to symptoms of heart failure. We did not find significant differences in the clinical characteristics of the patients with AS and CA or with CA only; however, this is possibly due to the relatively small sample size. Patients with AS and CA were older, but the $p$-value of the difference was only 0.055 .

At baseline echo, an aortic valve area less than 0.6 $\mathrm{cm}^{2}$ /body surface area (BSA) $\mathrm{m}^{2}$ was calculated in 3 cases, whereas in 2 cases, the calculated AVA was somewhat greater than $0.6 \mathrm{~cm}^{2} / \mathrm{BSA}\left(0.65\right.$ and $\left.0.63 \mathrm{~cm}^{2} / \mathrm{m}^{2}\right)-$ indicating moderate AS. In patients with an AVA/BSA less than $0.6 \mathrm{~cm}^{2}$, we performed dobutamine stress echo to distinguish between true-severe AS, and pseudosevere AS. One patient, with a left ventricular ejection fraction (LVEF) of $48 \%$, had true-severe AS: both echo and EKG suggested amyloidosis, CMR verified CA - and thus, the final diagnosis was AL amyloidosis (Table 2). This patient died within two months after diagnosis. In two patients, the stress test showed significant increase of AVA and thus provided evidence for pseudo-severe AS (Table 2) with a LVEF of 51 and $59 \%$. One of these patients was an 89-year old male patient, originally diagnosed with colon cancer - and heart failure revealed by preoperative evaluation. Echo showed LFLG AS and severe left ventricular hypertrophy with relative low voltage on 12-lead ECG. The dobutamine stress echo proved pseudo-severe AS, and the PYP isotope scan was typical for TTR CA. Finally, hemicolectomy was performed without any major complication. The cardiac images of this patient are shown in Fig. 1. The other patient with AL CA and pseudo-severe LFLG AS was started on therapy for plasma cell dyscrasia.

Apical sparing, the difference of the LS between the apical and mid + basal segments of the left ventricle was obvious at visual assessment in bull's eye images in all cases, but the ratio of average apical LS/(average basal LS + average mid LS) did not reach 1 .

\section{Discussion}

We found a $9 \%$ prevalence of moderate-to severe AS among consecutive, unselected CA patients. Of note, the vast majority of our CA patients had AL amyloidosis. This might be explained, in part, that our university hospital specializes also in hematology, in addition to 


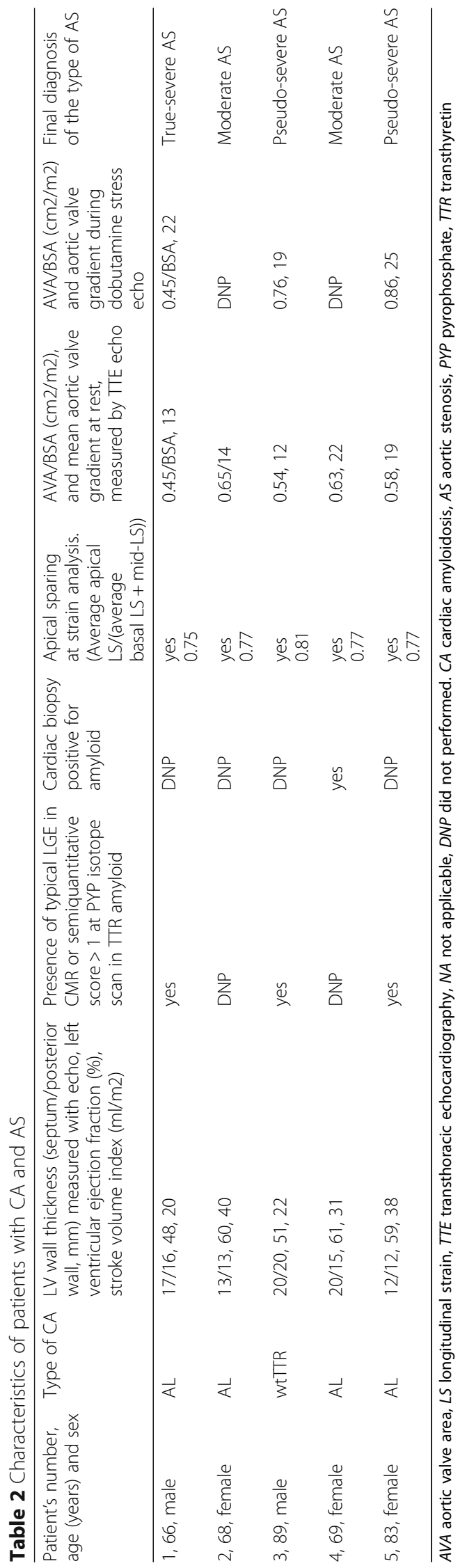




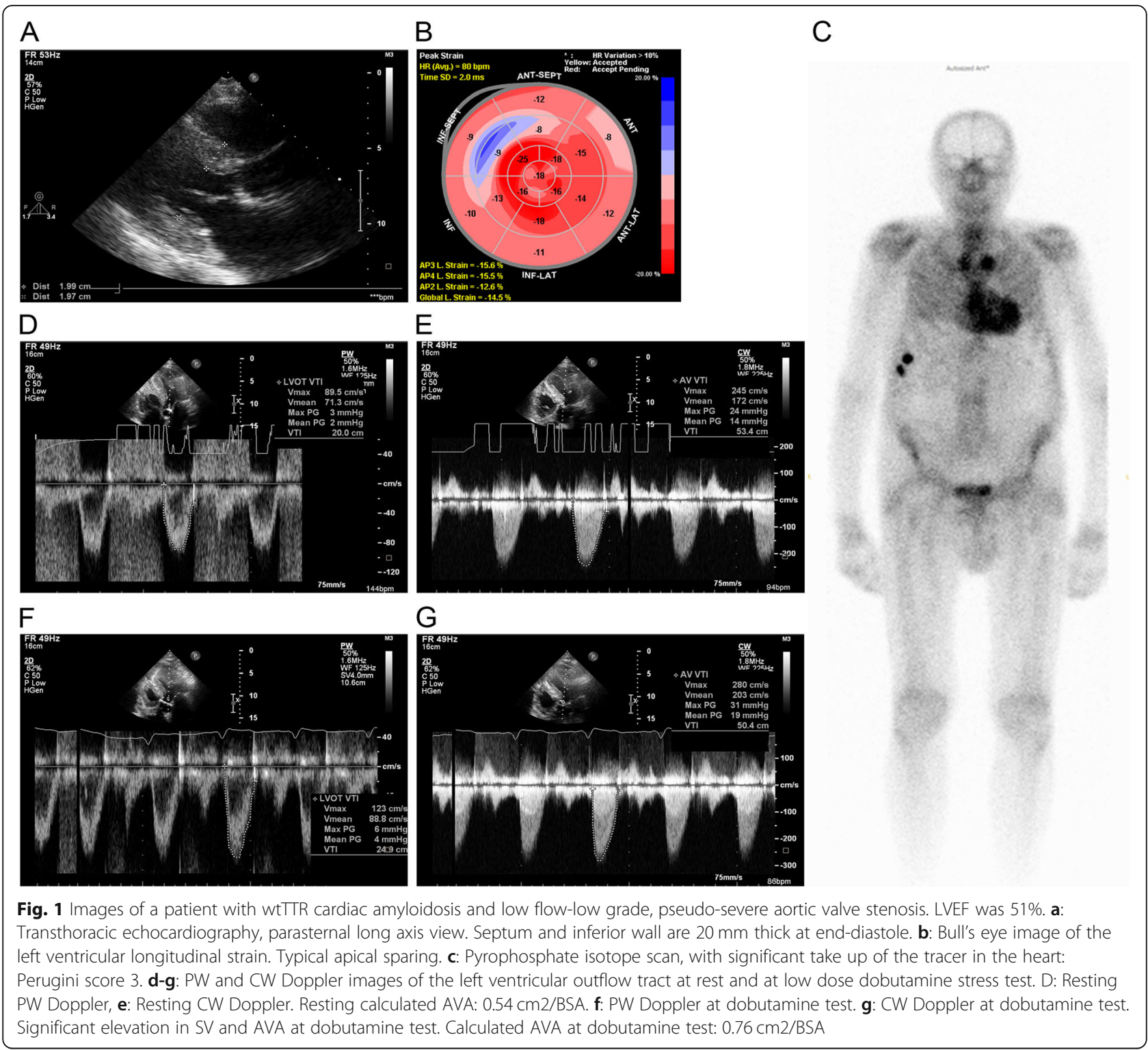

cardiology. Nowadays, the number of patients diagnosed with wild-type TTR cardiac amyloidosis is growing fast [3]. However, our patients were diagnosed between 2009 and 2019, and this might be the other cause of the relatively small percentage of TTR patients.

In previous years, the prevalence of CA in AS patients was explored using different methods and study designs and was found to be 4.1 [9] to $16 \%$ [6]. However, all the publications on these studies assert that these patients had TTR, but not AL amyloidosis. In two studies, where the prognosis was analyzed in addition, the authors found a significantly worse prognosis for patients with both AS and CA than for those with AS only. This was true after valve replacement [9], as well as when pooled patients with and without aortic valve replacement were studied [7]. On the other hand, the prevalence of AS among CA patients was studied only by a few authors; however, they also used registries of TTR CA patients. SPERRY et al. found that the prevalence of moderate to severe AS was $16 \%$ in TTR CA [11]. The retrospective analysis by JAVA et al. included patients with diverse causes of amyloidosis. They examined the results of aortic valve replacement in these patients [17]. The authors screened the database of Mayo Clinic (Rochester, MN, USA) for patients treated with aortic valve replacement and diagnosed with any type of amyloidosis in addition. Of the 16 patients meeting both criteria, only 6 had cardiac manifestations of amyloid disease; moreover, this subgroup of CA and AS patients was not analyzed separately, therefore this publication has a limited value for calculating the common prevalence of AS and CA. Based on these results, it appears that coexistence of TTR CA 
and AS is more common than that of AL CA and AS. A plausible explanation is the difference in the patients' age. The prevalence of AS is strongly age dependent, just as wtTTR amyloidosis $[2,3]$ is, and thus the risk of coexistence is also increasing with age. The median age of patients with AL amyloidosis is lower, as PCD affects younger patients. Thus the risk of a coincidence with AS is less.

Cardiac amyloidosis was once thought to be a very rare disease $[18,19]$. Studies in heart failure patients with preserved ejection fraction found CA to be surprisingly common, prevalence of CA was 17 and $29 \%$ in two different studies $[20,21]$. An autopsy study found a $25 \%$ prevalence of wtTTR cardiac amyloidosis over the age of 85 years [22]. The above-mentioned, recent publications on CA and AS also suggest that $\mathrm{CA}$ is more common than previously thought. This new piece of information has therapeutic implications, as well as diagnostic importance. Our patient population is rather small and therefore, it is not possible to identify and describe echocardiographic, laboratory, clinical, and prognostic differences between these patients with CA or CA and AS. The novelty of our retrospective analysis is that we found moderate-to severe AS to be relatively common in a CA population, where AL amyloidosis was present in $80 \%$.

The other novelty of our publication is, that it may draw attention to the use of dobutamine stress echo in LFLG AS patients with preserved ejection fraction in CA. Current guidelines do not recommend to perform dobutamine stress test with echo in LFLG AS patients with preserved ejection fraction (EF) [1]. The latest one by the European Society of Cardiology on the diagnosis and treatment of valvular heart disease [1] recognizes and underlines the diagnostic significance of LFLG AS. It recommends an integrative, stepwise diagnostic algorithm for these patients. First, the suspicion of AS should be raised by the morphology of the aortic valve. When the calculated aortic valve area (AVA) is less than $1 \mathrm{~cm}^{2}$, the mean pressure gradient is low $(<40 \mathrm{mmHg})$, and low-flow state is confirmed by the calculated lowstroke volume index $\left(<35 \mathrm{ml} / \mathrm{m}^{2}\right)$, the guideline recommends performing dobutamine stress echocardiography. This test can differentiate between "pseudo-severe AS", and true-severe AS. In pseudo-severe AS, AVA will increase together with stroke volume (SV), but the mean transvalvular gradient will not change significantly. In case of true-severe AS, the gradient will increase, but AVA will not change significantly. Of course, the dobutamine test will not aid the correct diagnosis in the absence of left ventricular contractile reserve - in this case, other clinical and imaging parameters should be taken into account. Interestingly, the guideline recommends performing the dobutamine test in LFLGAS only in patients with a reduced LVEF. From the pathophysiological perspective, this may seem reasonable, as one could speculate that only reduced LVEF can be increased by dobutamine. However, there are data on the usefulness and safety of this test in patients with LFLG AS, with reduced SV, but a normal EF. CAVAL et al [23] performed the dobutamine test in 55 patients with an average LVEF of $63 \%$ to differentiate true-severe AS from pseudosevere AS. They concluded that the method is useful and safe in these patients. We also performed this test in three cases with LFLG AS (LVEF being 48, 51, and 58\%), and it was diagnostic in all cases. In particular, the significant increase of AVA (Table 2) made it obvious that the symptoms of heart failure were caused by CA rather than pseudo-severe AS in two cases, and we found real, severe AS and CA in one case.

Although there are speculations that the pathomechanism of CA and AS may be connected, as amyloid deposition in the aortic valve could induce stenosis, there is no supporting evidence for that. Apparently, true-severe AS and CA coexist in a large number of patients. However, this can rather be explained simply by statistical distribution, as the prevalence of AS is quite high in the elderly, who also have a higher risk for wtTTR. In the above-mentioned studies, 6 to $16 \%$ of the AS patients systematically screened for TTR amyloidosis had wtTTR CA $[6,7,9,10]$. These patients were either not screened for AL amyloidosis, or AL CA was also excluded. On the other hand, the incidence of AS is not mentioned in a report published earlier on a large series of CA patients. In a study of 149 wtTTR CA patients, where authors focused on the echocardiographic, ECG, and clinical characteristics, there was not even a single patient identified with any degree of AS [24]. This may raise concerns about the validity of these data, and also suggests that the possible coexistence of these two clinical entities should always be considered - this is necessary for establishing the correct diagnosis. We suppose that the simultaneous presence of pronounced left ventricular hypertrophy and diastolic dysfunction is a possible reason for overlooking CA in AS. In particular, these abnormalities are common in both disorders, and LVH could be interpreted as the consequence of AS. On the other hand, it is easy to overlook AS in CA, as gradients can be surprisingly low due to the low stroke volume often seen in CA [25]. Precise calculation of AVA - and when necessary, dobutamine stress echo can clarify the diagnosis. Interestingly, we saw the phenomenon of "apical sparing" (preserved longitudinal endocardial strain in the apical versus basal segments) in all patients with CA and AS, which may be a useful prompt to look for CA when examining patients with AS. Of course, further "red flags" for CA should be evaluated, such as absolute or relative low voltage on the ECG, and other clinical signs of systemic amyloidosis. 
In our study, only one AL patient had significant, truesevere AS. This 66-year old patient with advanced (NYHA Class III) heart failure survived only 2 months after diagnosis. In this case, life expectancy was clearly limited by AS, CA, and the systemic disease of multiple myeloma. In such a case, it is not possible to determine the share of AS and CA in contributing to heart failure. In a UK study [9], patients with TTR CA and severe AS had a lot worse postoperative prognosis than those with calcified AS: the mortality rate during the follow-up period was $50 \%(3 / 6)$ versus $7.5 \%(8 / 106)$. These data suggest that when $\mathrm{CA}$ and AS coexist, both diseases contribute to the symptoms, as well as to poor prognosis.

SPERrY et al [11] at Cleveland Clinic Foundation (Ohio, USA) performed a retrospective analysis among 171 consecutive patients diagnosed with TTR CA at their institution. Diagnosis was based on endomyocardial biopsy, cardiac magnetic resonance, echocardiography (showing an apical sparing strain pattern), and/or $99 \mathrm{~m}-$ TcPYP scintigraphy. They compared the survival of patients with moderate (10 patients), low-flow, low-grade (11 patients), or high-grade (6 patients) AS to those without AS. Two-year mortality was high in both groups (37 and 33\%), but did not differ statistically, although 11 AS patients underwent aortic valve repair. The authors concluded that routine screening for TTR cardiac amyloidosis in LFLG AS patients might be useful, as it may detect the disease and influence therapy. This study, however, did not include AL CA patients. The ratio of AS patients who had dobutamine echocardiography is not mentioned and therefore, the number of patients with true-severe or pseudo-severe AS is not known. The authors highlight the role of echocardiography with strain analysis and that of $99 \mathrm{~m}$-TcPYP scintigraphy, but fail to mention the role of the dobutamine stress echo in the diagnostic process.

Based on our experience, we wish to underline the importance of dobutamine stress echocardiography in our LFLG AS population. This test can identify patients with true-severe AS, who may benefit from AVR or TAVI. We think that further stratification of LFLG AS, even in cases with preserved EF, is necessary in this patient population with $\mathrm{CA}$, to screen for patients, who may benefit from invasive treatment.

\section{Conclusion}

This is the first publication aimed to determine the prevalence of AS among unselected CA patients. In our patient population, the prevalence of moderate to severe AS was $9 \%$ based on resting echocardiography findings. Using dobutamine stress echocardiography, we were able to distinguish patients with pseudo-severe AS from those with true-severe AS, even in case of LFLGAS with preserved LVEF. Our results may draw attention to the coexistence of CA and AS, as well as to the importance of dobutamine echocardiography in determining the nature of AS in CA.

\section{Limitations}

The major limitation of our study is its small sample size. Because hematology is a major component of the clinical profile of the 3rd Department of Internal Medicine at Semmelweis University, our study population is not representative of CA patients in Hungary - those with AL amyloidosis are possibly overrepresented. Nevertheless, according to our knowledge, this study is the first to screen consecutive CA patients for AS.

\section{Abbreviations \\ AL amyloidosis: Light chain amyloidosis; AS: Aortic valve stenosis; AVA: Aortic valve area; BSA: Body surface area; CA: Cardiac amyloidosis; CMR: Cardiac magnetic resonance; EF: Ejection fraction; LFLG AS: Low flow-low grade aor- tic valve stenosis; LS: Longitudinal strain; LVEF: Left ventricular ejection fraction; LVH: Left ventricular hypertrophy; mTTR amyloidosis: Mutant type transthyretin amyloidosis; NYHA: New York Heart Association; PCD: Plasma cell dyscrasia; PYP: Pyrphoshate; TTR amyloidosis: Transthyretin amyloidosis; wtTTR: Wild-type transthyretin amyloidosis}

\section{Acknowledgements}

We thank Judit Kocsi for the technical assistance in the echo lab and Zsolt Förhécz for the critical help and advices.

\section{Authors' contributions}

ZP conceived and designed the retrospective analysis, looked over the echo images, and analyzed the data. GP wrote the draft manuscript, performed the statistics and collected data. ZJ collected clinical data and performed statistical analysis. SC performed and interpreted PYP scans and reviewed the manuscript. HV performed and interpreted the cardiac MRI studies and revised the manuscript. AA analyzed the data, wrote and reviewed the manuscript. ZProhászka performed the genetic analysis, helped to perform data collection and statistical analysis. TM and GV managed the diagnosis and therapy of AL amyloid patients, interpreted the clinical data and partially wrote the manuscript. All authors read and approved the final Manuscript.

\section{Funding}

There were no sources of funding.

Availability of data and materials

The authors declare that all data supporting the findings of this study are available within the article.

Ethics approval and consent to participate

The research was conducted ethically in accordance with the Declaration of Helsinki. The nature of this study is retrospective, and therefore retrospective ethics approval could not be obtained.

Consent for publication

We have the necessary consent from the patients who has individual personal images and data in the publication.

\section{Competing interests}

The authors declare that they have no competing interests.

\section{Author details}

13rd Department of Internal Medicine, Semmelweis University, Kútvölgyi 4 Budapest 1125, Hungary. ${ }^{2}$ Heart and Vascular Center, Semmelweis University, Városmajor 68, Budapest 1122, Hungary. ${ }^{3}$ Department of Nuclear Medicine, Semmelweis University, Üllői 78, Budapest 1082, Hungary. 
Received: 8 August 2019 Accepted: 18 December 2019

Published online: 26 December 2019

\section{References}

1. Baumgartner H, Falk V, Bax JJ, De Bonis M, Hamm C, Holm PJ, et al. 2017 ESC/EACTS guidelines for the management of valvular heart disease. Eur Heart J. 2017:38(36):2739-91.

2. Osnabrugge RL, Mylotte D, Head SJ, Van Mieghem NM, Nkomo VT, LeReun $\mathrm{CM}$, et al. Aortic stenosis in the elderly: disease prevalence and number of candidates for transcatheter aortic valve replacement: a meta-analysis and modeling study. J Am Coll Cardiol. 2013;62(11):1002-12.

3. Wechalekar AD, Gillmore JD, Hawkins PN. Systemic amyloidosis. Lancet. 2016:387(10038):2641-54

4. Salinger T, Hu K, Liu D, Herrmann S, Lorenz K, Ertl G, et al. Cardiac amyloidosis mimicking severe aortic valve stenosis - a case report demonstrating diagnostic pitfalls and role of dobutamine stress echocardiography. BMC Cardiovasc Disord. 2017;17(1):86.

5. Calero Nunez S, Tercero Martinez A, Garcia Lopez JC, Jimenez-Mazuecos J. Wild-type transthyretin-related cardiac amyloidosis and degenerative aortic stenosis: two inter-related pathologies in the elderly. Rev Esp Geriatr Gerontol. 2017;52(3):167-70.

6. Castano A, Narotsky DL, Hamid N, Khalique OK, Morgenstern R, DeLuca A, et al. Unveiling transthyretin cardiac amyloidosis and its predictors among elderly patients with severe aortic stenosis undergoing transcatheter aortic valve replacement. Eur Heart J. 2017;38(38):2879-87.

7. Cavalcante JL, Rijal S, Abdelkarim I, Althouse AD, Sharbaugh MS, Fridman Y, et al. Cardiac amyloidosis is prevalent in older patients with aortic stenosis and carries worse prognosis. J Cardiovasc Magn Reson. 2017;19(1):98.

8. Longhi S, Lorenzini M, Gagliardi C, Milandri A, Marzocchi A, Marrozzini C, et al. Coexistence of degenerative aortic stenosis and wild-type Transthyretin-related cardiac amyloidosis. JACC Cardiovasc Imaging. 2016; 9(3):325-7.

9. Treibel TA, Fontana M, Gilbertson JA, Castelletti S, White SK, Scully PR, et al. Occult Transthyretin Cardiac Amyloid in Severe Calcific Aortic Stenosis: Prevalence and Prognosis in Patients Undergoing Surgical Aortic Valve Replacement. Circ Cardiovasc Imaging. 2016;9(8).

10. Scully PR, Treibel TA, Fontana M, Lloyd G, Mullen M, Pugliese F, et al. Prevalence of cardiac amyloidosis in patients referred for Transcatheter aortic valve replacement. J Am Coll Cardiol. 2018;71(4):463-4.

11. Sperry BW, Jones BM, Vranian MN, Hanna M, Jaber WA. Recognizing Transthyretin cardiac amyloidosis in patients with aortic stenosis: impact on prognosis. JACC Cardiovasc Imaging. 2016;9(7):904-6.

12. Gillmore JD, Maurer MS, Falk RH, Merlini G, Damy T, Dispenzieri A, et al. Nonbiopsy diagnosis of cardiac Transthyretin amyloidosis. Circulation. 2016; 133(24):2404-12.

13. Lang RM, Bierig M, Devereux RB, Flachskampf FA, Foster E, Pellikka PA, et al. Recommendations for chamber quantification. Eur J Echocardiogr. 2006;7(2): 79-108.

14. Nagueh SF, Appleton CP, Gillebert TC, Marino PN, Oh JK, Smiseth OA, et al. Recommendations for the evaluation of left ventricular diastolic function by echocardiography. Eur J Echocardiogr. 2009;10(2):165-93.

15. Baumgartner H, Hung J, Bermejo J, Chambers JB, Evangelista A, Griffin BP, et al. Echocardiographic assessment of valve stenosis: EAE/ASE recommendations for clinical practice. Eur J Echocardiogr. 2009;10(1):1-25.

16. Phelan D, Collier P, Thavendiranathan P, Popovic ZB, Hanna M, Plana JC, et al. Relative apical sparing of longitudinal strain using two-dimensional speckle-tracking echocardiography is both sensitive and specific for the diagnosis of cardiac amyloidosis. Heart. 2012;98(19):1442-8.

17. Java AP, Greason KL, Dispenzieri A, Grogan M, King KS, Maleszewski JJ, et al. Aortic valve replacement in patients with amyloidosis. J Thorac Cardiovasc Surg. 2018;156(1):98-103

18. Kyle RA, Linos A, Beard CM, Linke RP, Gertz MA, O'Fallon WM, et al. Incidence and natural history of primary systemic amyloidosis in Olmsted County, Minnesota, 1950 through 1989. Blood. 1992:79(7):1817-22.

19. Pinney JH, Smith CJ, Taube JB, Lachmann HJ, Venner CP, Gibbs SD, et al. Systemic amyloidosis in England: an epidemiological study. Br J Haematol. 2013;161(4):525-32.

20. Bennani Smires $Y$, Victor G, Ribes D, Berry M, Cognet $T$, Mejean S, et al. Pilot study for left ventricular imaging phenotype of patients over 65 years old with heart failure and preserved ejection fraction: the high prevalence of amyloid cardiomyopathy. Int J Cardiovasc Imaging. 2016;32(9):1403-13.
21. Mohammed SF, Mirzoyev SA, Edwards WD, Dogan A, Grogan DR, Dunlay SM, et al. Left ventricular amyloid deposition in patients with heart failure and preserved ejection fraction. JACC Heart Fail. 2014;2(2):113-22.

22. Tanskanen M, Peuralinna T, Polvikoski T, Notkola IL, Sulkava R, Hardy J, et al. Senile systemic amyloidosis affects $25 \%$ of the very aged and associates with genetic variation in alpha2-macroglobulin and tau: a population-based autopsy study. Ann Med. 2008;40(3):232-9.

23. Clavel MA, Ennezat PV, Marechaux S, Dumesnil JG, Capoulade R, Hachicha Z et al. Stress echocardiography to assess stenosis severity and predict outcome in patients with paradoxical low-flow, low-gradient aortic stenosis and preserved LVEF. JACC Cardiovasc Imaging. 2013;6(2):175-83.

24. Gonzalez-Lopez E, Gagliardi C, Dominguez F, Quarta CC, de Haro-Del Moral FJ, Milandri A, et al. Clinical characteristics of wild-type transthyretin cardiac amyloidosis: disproving myths. Eur Heart J. 2017;38(24):1895-904.

25. Flodrova P, Flodr P, Pika T, Vymetal J, Holub D, Dzubak P, et al. Cardiac amyloidosis: from clinical suspicion to morphological diagnosis. Pathology. 2018;50(3):261-8

\section{Publisher's Note}

Springer Nature remains neutral with regard to jurisdictional claims in published maps and institutional affiliations.

\section{Ready to submit your research? Choose BMC and benefit from:}

- fast, convenient online submission

- thorough peer review by experienced researchers in your field

- rapid publication on acceptance

- support for research data, including large and complex data types

- gold Open Access which fosters wider collaboration and increased citations

- maximum visibility for your research: over $100 \mathrm{M}$ website views per year

At BMC, research is always in progress.

Learn more biomedcentral.com/submissions 